\title{
Investigating Absolute Stereochemical Configuration with Coulomb Explosion Imaging
}

\author{
Martin Pitzer ${ }^{\star a b}$, Robert Berger ${ }^{c}$, Jürgen Stohnerd, Reinhard Dörnera , and Markus Schöfflerª
}

\begin{abstract}
It is a particularly challenging task in stereochemistry to determine the absolute configuration of chiral molecules, i.e. to assign to a given sample the microscopic enantiomeric structure. In recent years, Coulomb Explosion Imaging (CEI) has been shown to yield directly the absolute configuration of small molecules in the gas phase. This contribution describes the experimental basics of this approach, highlights the most significant results and discusses limitations. A short discussion on extending Coulomb Explosion Imaging beyond analytic aspects to fundamental questions of molecular chirality concludes this review.
\end{abstract}

Keywords: Absolute configuration · Chirality · Coincidence $\cdot$ Gas-phase $\cdot$ Photoionisation
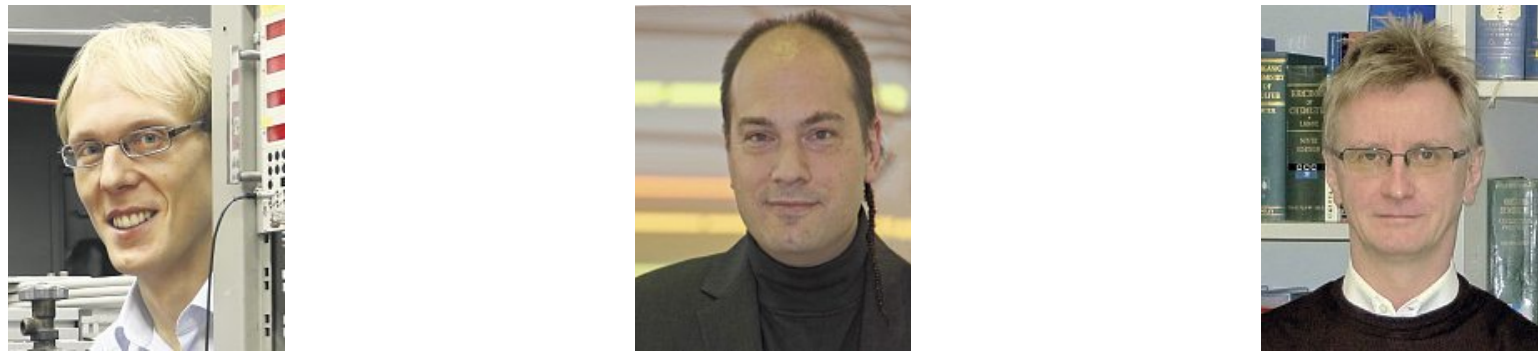

Martin Pitzer studied Physics at the Karlsruhe Institute of Technology (KIT) and the Ecole Polytechnique Fédérale de Lausanne. In 2015, he obtained his $\mathrm{PhD}$ from the University of Frankfurt, having worked on coincidence experiments with chiral molecules in the group of Reinhard Dörner. After an intermezzo as an independent junior researcher at the University of Kassel, he is currently investigating cold molecular collisions in the group of Ed Narevicius at the Weizmann Institute of Science. (Photo: Uwe Dettmar)

\footnotetext{
$\overline{{ }^{*} \text { Correspondence: }}$ Dr. M. Pitzerab, Dr. M. Schöffler E-mail: martin.pitzer@weizmann.ac.il; schoeffler@atom.uni-frankfurt.de alnstitut für Kernphysik, Goethe-Universität Max-von-Laue-Str. 1, 60438 Frankfurt, Germany bDepartment of Chemical and Biological Physics Weizmann Institute of Science P. O. Box 26, 76100 Rehovot, Israel 'Philipps-Universität Marburg, Fachbereich Chemie Hans-Meerwein-Straße, 35032 Marburg, Germany d Zürcher Hochschule für Angewandte Wissenschaften, Campus Reidbach

Institut für Chemie und Biotechnologie

Einsiedlerstrasse 31, CH-8820 Wädenswil
}

Robert Berger studied in Berlin and Münster. After receiving his $\mathrm{PhD}$ for his work on theoretical spectroscopy in the group of Martin Klessinger, he spent a postdoctoral stay at ETH Zurich with Martin Quack. He returned with a Liebig fellowship to TU Berlin, where Helmut Schwarz was his mentor. Robert Berger was awarded subsequently a junior research group by the VolkswagenFoundation and became fellow of the Frankfurt Institute for Advanced Studies. After an appointment as associate professor at TU Darmstadt, he became full professor for Theoretical Chemistry at Philipps-Universität Marburg. (Photo: Sebastian Marquardt)
Jürgen Stohner studied Chemistry in Tübingen; he continued his studies in Chemistry at the ETH Zürich, where he received his $\mathrm{PhD}$ in 1994 on quantum dynamics and IR multiphoton excitation in molecules with low symmetry in the research group of Prof. Quack. After a postdoc with Prof. Carrington at the Université de Montréal he returned to ETH. Since 1998 he is lecturer at the Zürich University of Applied Sciences (formerly Technikum Winterthur) and was promoted to Professor ZFH in 2009. His research interests range from $a b$ initio calculations, high-resolution (IR) spectroscopy, vibrational circular dichroism to the synthesis and enantio-separation of small chiral molecules relevant for studying chiroptical properties. He is currently Chair of the IUPAC Interdivisional Commission on Terminology, Nomenclature and Symbols (ICTNS). His last IUPAC project as Task Group Chair was concerned with the redefinition of the mole, which will eventually find its way into the new SI to be launched in 2019. 


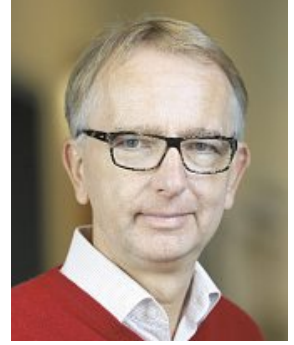

Reinhard Dörner studied Physics at Goethe University Frankfurt where he obtained his $\mathrm{PhD}$ in the group of Horst Schmidt-Böcking. He did a postdoc at LBNL Berkeley, USA. Since 2002 he is a full professor at the physics department of Goethe University Frankfurt am Main. His main research interests are multicoincidence experiments in atomic and molecular physics using lasers, synchrotrons and charged particle beams. (Photo: Uwe Dettmar)

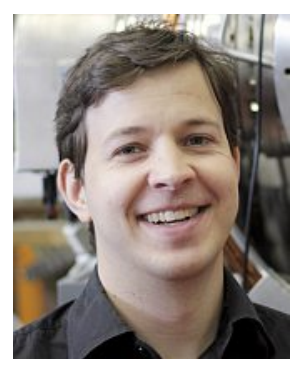

Markus Schöffler studied Physics at the Goethe-University in Frankfurt where he obtained his $\mathrm{PhD}$ in 2006. He was FeodorLynen fellow of the Humboldt society at the Advanced Light Source in Berkeley, California and post-doctoral fellow at the Technical University in Vienna. Since 2011 he has been working as group leader at the Goethe-University, with a focus on probing chiral molecules with coincidence techniques. His research was awarded with the Adolf-Messer-Stiftungspreis in 2014 and with the Röntgenpreis of the University of Gießen in 2016. (Photo: Claudia Freudenberger)

\section{Introduction - The Quest for Absolute Configuration}

In 1891, Emil Fischer published an important result of his efforts to determine the molecular configuration of optically active sugar compounds. [1] Following various chemical relations, he could narrow the possible configurations of a molecule he called Zuckersäure (now known as saccharic acid), to two mirror image structures. However, he had no possibility to find out which of these two spatial arrangements corresponded to the D-saccharic acid.
This assignment proved to be surprisingly difficult, so that only relative configurations could be determined for more than 50 years. In 1951, Bijvoet et al. determined the absolute configuration of a double salt of tartaric acid using anomalous X-ray diffraction. ${ }^{[2]}$

Until today, this technique and its modifications are the only methods routinely used to directly determine absolute configuration, i.e. without additional input from theory. X-ray diffraction, however, usually requires a (micro)crystalline sample and is thus not suitable for all species.

\section{Determining Molecular Structure with Coulomb Explosion Imaging}

A completely different way to determine bond lengths and molecular structures for small molecules in the gas phase emerged in the 1970s in the field of accelerator physics: At the beginning, molecular ions such as $\mathrm{HeH}^{+}$or $\mathrm{H}_{3}^{+}$were accelerated to kinetic energies of several millions of electron volts $(\mathrm{MeV})$, corresponding to velocities of around $10000 \mathrm{~km} / \mathrm{s}$ or a few atomic units of velocity. ${ }^{[3]}$ These species traversed a thin polymer foil of less than a micrometre thickness, stripping off the electrons quasi-instantaneously. The electrostatic repulsion of the remaining positively charged cores led to small deviations in the trajectories that were detected a few metres downstream. Since the structural information was inferred from the re- pulsive Coulomb interaction, the method was coined Coulomb Explosion Imaging (CEI).

Subsequently, CEI was extended to more complex species and allowed to determine the structure of species such as small cationic carbon clusters ${ }^{[4]}$ and $\mathrm{CH}_{4}{ }^{+}{ }^{[5]}$ It was assumed that also the absolute configuration of chiral molecules could be determined that way. Due to limitations in the acceleration voltage, however, molecules with molar mass $M>50 \mathrm{~g} /$ mol could not be accelerated to sufficiently high kinetic energies to induce efficient charge stripping.

In the last two decades, the COLTRIMS (Cold Target Recoil Ion Momentum Spectroscopy) or 'Reaction Microscope' approach has been established and found widespread application in atomic and molecular physics. ${ }^{[6]}$ Fig. 1 shows the schematics of this method.

The sample expands as a supersonic gas jet into a vacuum chamber (in Fig. 1 from below) where the neutral molecules intersect with ionising radiation, such as femtosecond lasers, synchrotron radiation or fast ions. As in the foil-induced technique, the multiply ionised molecule undergoes Coulomb explosion, driving the fragment cations apart. They are guided by electrostatic fields towards a time- and position-sensitive detector. Similar to a conventional Time-of-Flight (TOF) mass spectrometer, the arrival time allows the mass-to-charge ratio of the cations to be identified. There is, however, an important

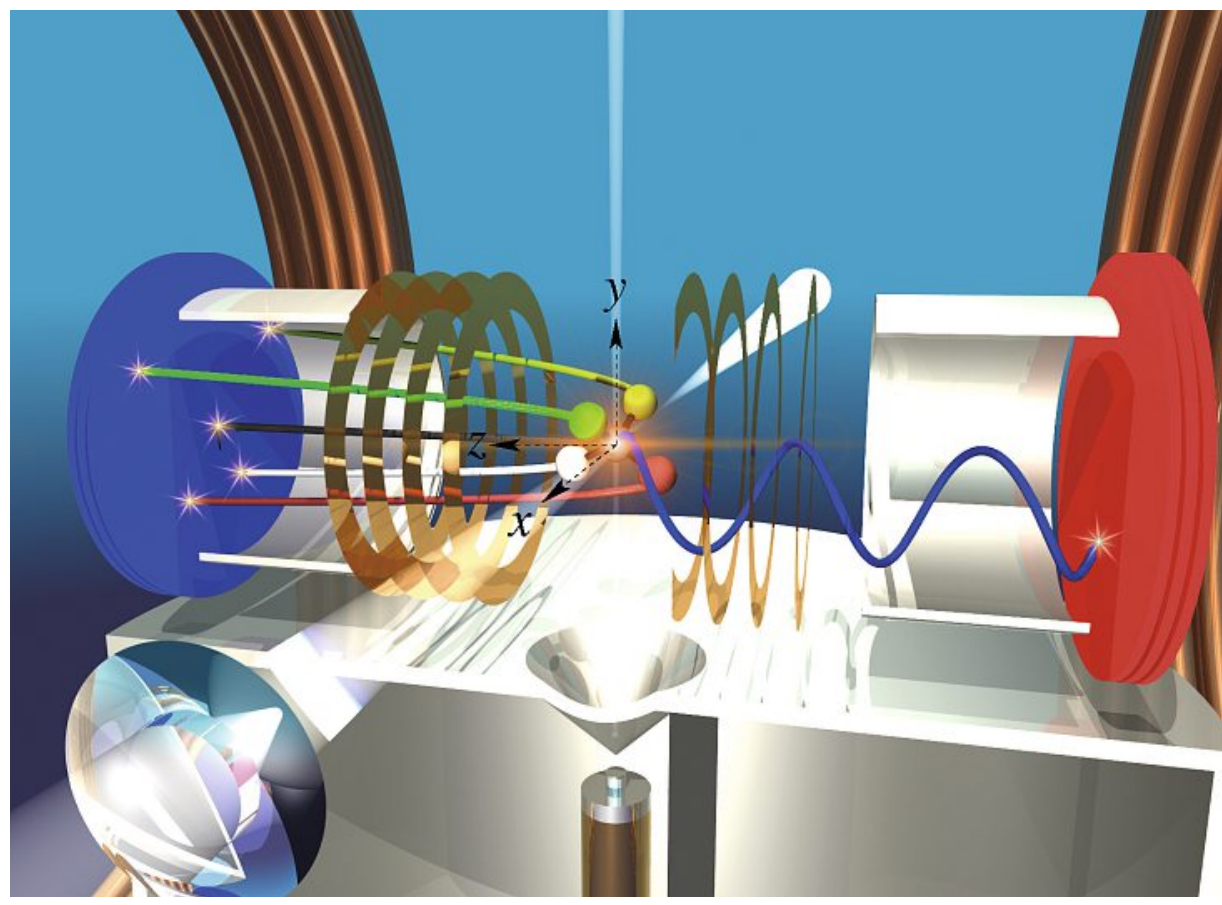

Fig. 1: Schematics of the COLTRIMS technique. The gas jet (from below) intersects with ionising radiation; cations are guided by the electrostatic field to the left, electrons to the right (only one electron trajectory shown for clarity). The single-particle detectors are time- and position-sensitive so that the trajectories of all charged fragments - and thus their initial velocities - can be reconstructed. Figure reproduced from ref. [10] (Supplementary Material) with permission by AAAS. 
difference: Most TOF setups are designed to make the time-of-flight independent of the initial velocity of the particles to obtain narrow peaks in the TOF spectrum and thus a high mass resolution. In a COLTRIMS setup, on the contrary, low electric fields are used so that the velocity (magnitude and direction) of the fragment cation affects the arrival time. In addition, the detectors record the position of the cation impact. These three coordinates (time-offlight and two-dimensional position) allow reconstruction of the three-dimensional linear momentum vector that the particles obtain in the Coulomb Explosion (for more details, see e.g. ref. [7]).

If the particle density in the gas jet is so low that not more than one molecule is fragmented per ionising (laser) shot, the momenta of the fragments can be recorded in coincidence. With these data, correlations between the fragment cations can be inferred for each measured molecule.

\section{Finding the Absolute Configuration of Single Molecules}

Only in 2013, both approaches to Coulomb Explosion Imaging - foil-induced and light-induced - were successfully used by two independent groups to distinguish enantiomers and infer the absolute configuration of small molecules. One group applied the previously described stripping-foil technique to a chiral epoxide (2,3-dideuterooxirane) with a nominal molar mass of $M=46 \mathrm{~g} / \mathrm{mol}^{\left[{ }^{[8]}\right.}$ The configuration could be determined with high statistical significance and chemically related to the optical rotation of glyceraldehyde. ${ }^{[9]}$ However, the supposedly enantiopure sample showed an enantiomeric excess of only $34 \%$ in the data.

The authors of the present contribution and their colleagues used a COLTRIMS setup to record all five atomic cations of bromochlorofluoromethane $(\mathrm{CHBrClF})$ after ionisation with a femtosecond (fs) laser pulse.[10] This prototypical chiral species is particularly suited for gas phase experiments, due to its simplicity but also due to a relatively high vapour pressure of about $500 \mathrm{hPa}$.

In ref. [10], racemic $\mathrm{CHBrClF}$ was synthesised following the protocol of Hine et al..$^{11]}$ by fluorinating $\mathrm{CHBr}_{2} \mathrm{Cl}$ with $\mathrm{HgF}_{2}$. Doyle and $\operatorname{Vog}{ }^{[12]}$ as well as Beil et al. ${ }^{[1]}$ obtained racemic $\mathrm{CHBrClF}$ starting from chlorotrifluoroethylene. We synthesised the target compound additionally by starting from the symmetric 1,2-dichloro-1,2-difluoroethylene, which has a much higher boiling point and, due to its symmetric structure, leads to fewer byproducts via the racemic acetic acid $\mathrm{CBrClFCOOH}$, which was enantio-separated either by fractional crystallisation with strychnine to yield $\mathrm{CHBrClF}$ after decarboxylation under retention of configuration or by preparative chiral gas-chromatography of the decarboxylated racemic halomethane, $\mathrm{CHBrClF}$, with a chiral stationary phase. ${ }^{[14]}$ Gas-chromatographic resolution of enantiomers of small halomethanes $(\mathrm{CHBrClF}$, $\mathrm{CHClFI}, \mathrm{CF}_{3} \mathrm{CHBrCl}$ ) was achieved in the past only on an analytical scale using commercially available chiral stationary phases. ${ }^{[13,15]}$ A much more versatile and efficient synthesis has been described recently by starting from diethyl malonates. ${ }^{[16]}$

The racemic $\mathrm{CHBrClF}$ was expanded by virtue of its room-temperature vapour pressure (without carrier gas) into the spectrometer and intersected with the laser. The high intensity of the laser pulse (around $6 \cdot 10^{14} \mathrm{~W} / \mathrm{cm}^{2}$ ) can lead to up to fivefold ionisation and fragmentation into singly charged cations.

In analogy to the structural arrangement, the linear momentum vectors measured in coincidence are used to determine the molecular absolute configuration according to the Cahn-Ingold-Prelog (CIP) rules. Fig. 2 shows the distribution of $\cos (\theta)$ from a racemic mixture where $\cos (\theta)=\boldsymbol{p}_{\mathrm{F}} \cdot\left(\boldsymbol{p}_{\mathrm{Cl}} \times \boldsymbol{p}_{\mathrm{Br}}\right) \cdot\left(\left|\boldsymbol{p}_{\mathrm{F}}\right| \cdot\left|\boldsymbol{p}_{\mathrm{Cl}} \times \boldsymbol{p}_{\mathrm{Br}}\right|\right)^{-1}$ depends on the triple product between the different linear momentum vectors $p_{i}$. If the linear momentum vectors of bromine, chlorine and fluorine are oriented in an $\mathrm{S}$-type configuration, the triple product is negative; for an R-type configuration, it is positive. As expected for a racemate, two peaks with equal intensity can be seen; the fact that almost no background is present indicates that the enantiomer assignment is reliable on a single molecule level. The used sample contained all isotopes in natural abundance which was confirmed in the sum momentum spectrum; ${ }^{[10]}$ for Figs. 2 and 3 , only the isotopic composition ${ }^{12} \mathrm{CH}^{79} \mathrm{Br}^{37} \mathrm{Cl}^{19} \mathrm{~F}$ was selected.
Since only the relative orientations play a role here, the random orientation of the gas-phase molecules does not preclude the determination of absolute configuration. Light-induced Coulomb Explosion Imaging is also applicable to determine the absolute configuration of molecules that are chiral due to isotopic substitution as has been demonstrated in ref. [10] for $\mathrm{CHBr}^{37} \mathrm{Cl}^{35} \mathrm{Cl}$, which is contained as a racemic mixture in a sample of $\mathrm{CHBrCl}_{2}$ with isotopes in natural abundance.

How good is the assumption that the measured momenta represent the initial structure? For diatomics even the quantum limit of measurement has been reached[17] and for triatomic systems, high accuracy in structure determination has been achieved for $\mathrm{He}_{3}{ }^{[18]}$ For larger molecules, however, this is less clear. Classical molecular dynamics simulations suggest that the final momenta reproduce the angles of the initial spatial structure with deviation of only a few degrees in the case of $\mathrm{CHBrClF}$. This is confirmed by the experimental findings: Fig. 3 shows the measured linear momenta - rotated a posteriori to a molecular frame of reference - overlaid with the molecular equilibrium structure. The linear momenta of the halogens are well located, indicating that the assignment of enantiomers via the fragment momenta is indeed valid. The proton (shown in white), however, follows a broader distribution. Bearing in mind the period of the $\mathrm{CH}$ stretch of around $10 \mathrm{fs}$, we assume that the (vibrational) motion of the proton in the molecular potential is of the same order as the laser pulse ( $40 \mathrm{fs})$. This could be one of the reasons for the observed broadening. Possible additional effects are the width of the ground state wavefunction (which is largest for the light proton), bending modes and the interaction of the proton with the laser field during and after the ionisation itself.

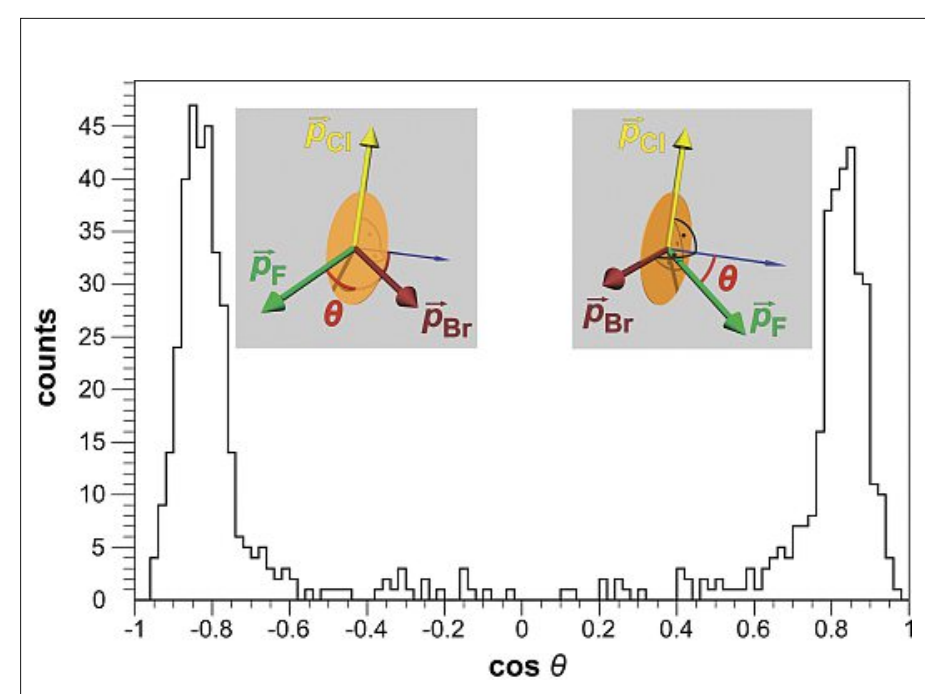

Fig. 2. The chirality parameter $\cos (\theta)$ as defined in the text, for the ionisation of $\mathrm{CHBrClF}$ with femtosecond laser pulses. The inset shows the graphical interpretation of cos $(\theta)$. As is expected for a racemic mixture, the abundance for the two enantiomers is equal within the statistical uncertainty. The low background indicates a highly reliable assignment. Figure reproduced from ref. [10] with permission by AAAS. 


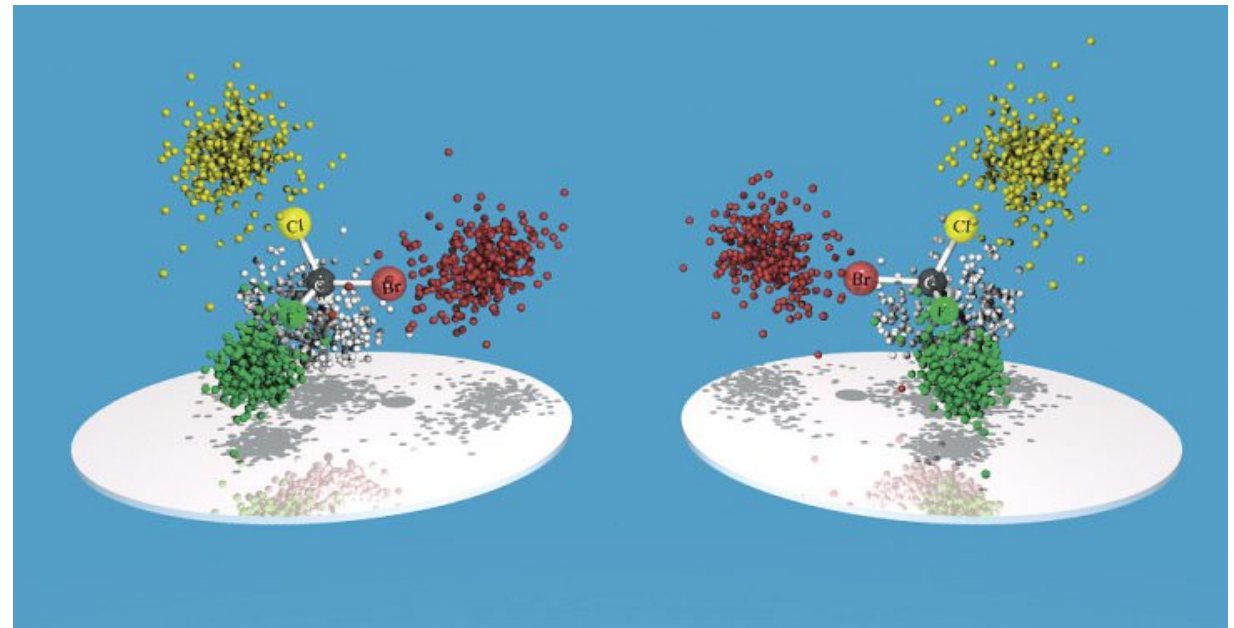

Fig. 3. Three-dimensional representation of the linear momenta in the molecular frame for the fragmentation into five atomic ions (laser excitation). The momenta are rotated to overlay with a structure model. Colour codes are white: H; black: C; green: F; yellow: $\mathrm{Cl}$; red: Br. For better visibility, hydrogen moments are expanded by a factor of 2. Reproduced from ref. [25]. CC BY 3.0.

\section{Fragmentation Timescale: Femtosecond Laser and Single X-ray Photons}

Replacing the proton by a deuteron is expected to slow down vibrational motion and might lead thus to a better defined momentum distribution. Measurements on isotopically chiral $\mathrm{CDFCl}_{2}$ did, however, show an almost identical distribution for the two isotopomers (see Fig. 4).

A faster and 'cleaner' ionisation can be achieved with single-photon excitation: By absorbing an X-ray photon, a core or inner valence hole in one of the atoms can be created; the subsequent electronic relaxation processes lead (with a certain branching ratio), to four or five-fold ionisation as well. Since these electronic processes are usually faster than nuclear dynamics (cf. Born-Oppenheimer approximation), the molecular structure is expected to be essentially 'frozen' during the process of multiple ionisation. Results on the configuration of $\mathrm{CHBrClF}$ after excitation with photons from a synchrotron source (photon energy: $710 \mathrm{eV}$ ) show that the proton distribution is indeed narrower. ${ }^{[19]}$
Fig. 4: Separation of enantiomers of isotopically chiral dichlorofluoromethane after ionisation with a femtosecond laser pulse, using the measured proton momenta (blue) and the measured deuteron momentum (red) respectively. The chirality parameter is defined as $\cos \left(\theta^{\prime}\right)$ $=\boldsymbol{p}_{\mathrm{H}} \bullet\left(\boldsymbol{p}_{35 \mathrm{Cl}} \times \boldsymbol{p}_{37 \mathrm{Cl}}\right)$. $\left(\left|\boldsymbol{p}_{\mathrm{H}}\right| \cdot\left|\boldsymbol{p}_{35 \mathrm{Cl}} \times \boldsymbol{p}_{37 \mathrm{Cl}}\right|\right)^{-1}$ for $\mathrm{CHFCl}_{2}$ and cos $\left(\theta^{\prime}\right)=\boldsymbol{p}_{\mathrm{D}} \bullet\left(\boldsymbol{p}_{35 \mathrm{Cl}} \times \boldsymbol{p}_{37 \mathrm{Cl}}\right)$. $\left(\left|\boldsymbol{p}_{\mathrm{D}}\right| \cdot\left|\boldsymbol{p}_{35 \mathrm{Cl}} \times \boldsymbol{p}_{37 \mathrm{Cl}}\right|\right)^{-1}$ for $\mathrm{CDFCl}_{2}$. Despite its lower vibrational frequency, the deuterated species does not show a narrower distribution.

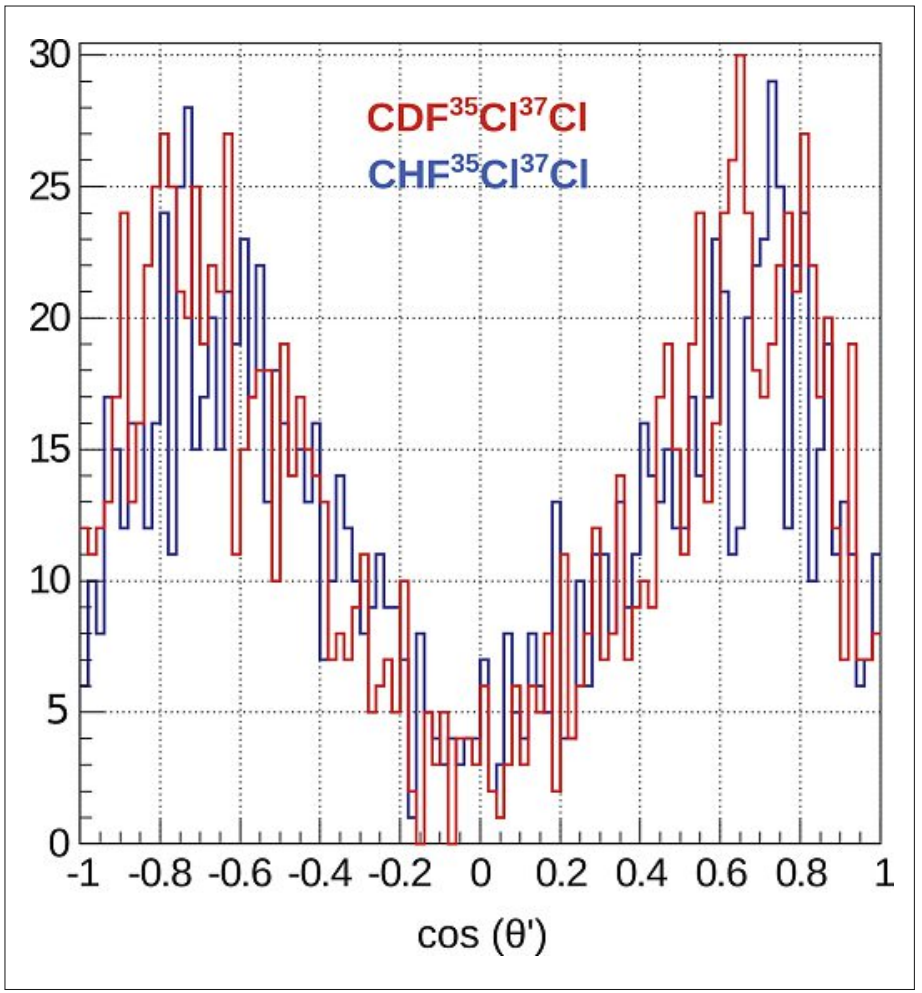

\section{Prospects and Limitations for Larger Molecules}

With only atomic ligands bound to the chiral centre, $\mathrm{CHBrClF}$ is one of the molecules where Coulomb Explosion Imaging is expected to yield the clearest results. How well can the measured momenta reproduce the configuration if the molecules get more complex? In this case, the complete fragmentation into all constituent atoms is very improbable and difficult to analyse. It is thus necessary to explore to what extent molecular cationic fragments reveal information about the configuration. A first step was performed recently by recording the Coulomb Explosion of a racemic mixture of halothane (2-bromo-2-chloro-1,1,1-trifluoroethane, $\left.\mathrm{CHBrClCF}_{3}\right)_{.}{ }^{[20]}$ One of the fragmentation pathways yielded the fragments $\mathrm{CH}^{+}$, $\mathrm{Cl}^{+}, \mathrm{Br}^{+}$and $\mathrm{CF}_{3}^{+}$. Although there is some background present, a good separation of enantiomers can be observed.

It is expected that Coulomb Explosion Imaging is likely to fail in assigning absolute configuration of larger molecules, especially for those with several stereocentres. Another drawback for large molecules is the decreasing branching ratio for those channels that allow determining configuration: As the ionisation and subsequent fragmentation are statistical processes, most of the excited molecules decay into two or three fragments. Since at least three linearly independent momentum vectors are necessary to determine a 3D configuration, channels with less than four fragments cannot be used for configuration assignment. Already for the molecules discussed here, the share of useful fragmentations is below $10^{-4}$ of the total number of ionisation events; since the molecular fragmentations have to be recorded one by one to ensure the coincidence, the reported measurements took 10 hours or more. A major step forward is made here by using detectors with higher efficiencies, which can speed up this process by at least an order of magnitude; ${ }^{[21]}$ the rapid development of lasers with higher repetition rates $(\mathrm{MHz})$ and sufficient pulse energy $(10 \mu \mathrm{J})$ will likely decrease measurement time even more.

The rather inefficient fragmentation is also the main reason that rather large amounts of sample have been used in our measurements so far (around $1 \mathrm{~g}$, or several $10 \mathrm{~s}$ of mmol). As a consequence, experiments on chiral molecules with COLTRIMS have so far been restricted to racemic mixtures. It is thus an upcoming task for this technique to relate the absolute configuration measured with CEI to chiroptical properties of an enantioenriched or enantiopure sample. 


\section{Electron Spectroscopy in the Molecular Frame}

Besides the application for stereochemical analysis of chiral samples, Coulomb Explosion Imaging offers the possibility to investigate chirality at a single-molecule level.

A COLTRIMS apparatus is also capable of detecting electrons in coincidence with the cations: If a second time- and position-sensitive detector is mounted to the other side of the spectrometer, electrons are guided to there by the electrostatic field, and their impact positions allow to reconstruct electron energies and emission angles as well (with a few complications, $c f$. ref. [6]). This approach is routinely used in many laboratories to investigate molecular ionisation processes of small molecules by single photons, free electron lasers and femtosecond lasers.

In the case of stereochemistry, it might lead to a better understanding of the intriguing interplay between chiral (i.e. circularly polarised) light and chiral molecules. Photo-electron circular dichroism (PECD), i.e. the angular asymmetry of photoelectrons with respect to the circularly polarised light beam, is increasingly used to study chirality. ${ }^{[22]}$ These measurements are usually performed on a randomly oriented sample of molecules in the gas phase. The coincident detection of electrons and cations allows studying PECD in the molecular frame. Observing the breakup of enantiopure methyloxirane $\left(\mathrm{C}_{3} \mathrm{H}_{6} \mathrm{O}\right)$ into two fragments can provide a molecular axis; the strength of the forward-backward asymmetry has thus been related to the molecular orientation. ${ }^{[23]}$

Coulomb Explosion Imaging and related coincidence techniques allow investigation of chiral molecules with an unprecedented level of detail. In addition to providing information about the molecular configuration, it might in the future contribute to a better understanding of the dynamics of chiral molecules and their interaction with chemical or physical probes. In particular, observing vector correlations as is possible with COLTRIMS might be a new avenue to investigate fundamental symmetry properties such as parity violation. ${ }^{[24]}$

\section{Acknowledgements}

We are grateful to all our colleagues without whom these demanding experiments would not have succeeded, especially Maksim Kunitski, Till Jahnke, and Lothar Schmidt as well as Michael Reggelin, Alexander Schießer, Sebastian Marquardt and Sabrina Marquardt. We thank Horst Schmidt-Böcking for inspiring and fruitful discussions. The work was supported by the State of Hesse Initiative for the Development of Scientific and Economic Excellence (LOEWE) within the focus-project Electron Dynamics of Chiral Systems (ELCH) and by the Deutsche Forschungsgemeinschaft via Sonderforschungsbereich 1319 (ELCH). We thank the staff of Synchrotron SOLEIL for running the facility and providing beamtimes under projects 20140056 and 20141178 and especially SEXTANTS beamline for their excellent support. M. S. gratefully acknowledges support by the Adolf Messer foundation. J. S. gratefully acknowledges present and past group members who contributed to the progress of our work, namely V. Galati, S. Manov, M. Mazenauer, and B. Spenger. Our work was supported financially by ZHAW grants ASF2016 and ASF-2017.

Received: April 10, 2018

[1] E. Fischer, Ber. dt. chem. Ges. 1891, 24, 1836. [2] J. M. Bijvoet, A. F. Peerdeman, A. J. van Bommel, Nature 1951, 168, 271.

[3] a) Z. Vager, D. S. Gemmell, B. J. Zabransky, Phys. Rev. A 1976, 14, 638; b) M. J. Gaillard, D. S. Gemmell, G. Goldring, I. Levine, W. J. Pietsch, J. C. Poizat, A. J. Ratkowski, J. Remillieux, Z. Vager, B. J. Zabransky, Phys. Rev. A 1978, 17, 1797.

[4] H. Feldman, D. Kella, E. Malkin, E. Miklazky, Z Vager, J. Zajfman, R. Naaman, J. Chem. Soc. Faraday Trans. 1990, 86, 2469.

[5] Z. Vager, E. P. Kanter, G. Both, P. J. Cooney, A Faibis, W. Koenig, B. J. Zabransky, D. Zajfman, Phys. Rev. Lett. 1986, 57, 2793.

[6] a) R. Dörner, V. Mergel, O. Jagutzki, L. Spielberger, J. Ullrich, R. Moshammer, H. Schmidt-Böcking, Phys. Rep. 2000, 330, 95; b) J. Ullrich, R. Moshammer, A. Dorn, R. Dörner, L. P. H. Schmidt, H Schmidt-Böcking, Rep. Prog. Phys. 2003, 66, 1463.

[7] a) M. Pitzer, K. Fehre, M. Kunitski, T. Jahnke, L. Schmidt, H. Schmidt-Böcking, R. Dörner, M. Schöffler, J. Visualized Exp. 2017, 126, e56062 b) M. Pitzer, J. Phys. B: Opt. At. Mol. Phys. 2017, 50, 153001

[8] P. Herwig, K. Zawatzky, M. Grieser, O. Heber, B. Jordon-Thaden, C. Krantz, O. Novotny, R. Repnow, V. Schurig, D. Schwalm, Z. Vager, A Wolf, O. Trapp, H. Kreckel, Science 2013, 342 , 1084.

[9] K. Zawatzky, P. Herwig, M. Grieser, O. Heber, B. Jordon-Thaden, C. Krantz, O. Novotny, R. Repnow, V. Schurig, D. Schwalm, Z. Vager, A. Wolf, H. Kreckel, O. Trapp, Chem. - Eur. J. 2014, 20, 5555.

[10] M. Pitzer, M. Kunitski, A. S. Johnson, T. Jahnke, H. Sann, F. Sturm, L. P. H. Schmidt, H. Schmidt-Böcking, R. Dörner, J. Stohner, J. Kiedrowski, M. Reggelin, S. Marquardt, A. Schießer, R. Berger, M. S. Schöffler, Science 2013, 341, 1096

[11] J. Hine, A. M. Dowell, J. E. Singley, J. Am. Chem. Soc. 1956, 78, 479.

[12] T. R. Doyle, O. Vogl, J. Am. Chem. Soc. 1989, $111,8510$.

[13] A. Beil, D. Luckhaus, M. Quack, Ber. Bunsenges. Phys. Chem. 1996, 100, 1853.

[14] a) B. Spenger, J. Stohner, Europ. Patentamt, patent EP 3069777 A1; b) S. Manov, V. Galati, M. Meister, M. Mazenauer, B. Spenger, J. Stohner, Proc. XXth Symp. At., Cluster and Surf. Phys. (SASP) 2016, 199
[15] a) W. A. König, 'Gas Chromatographic Enantiomer Separation with Modified Cyclodextrins', Hüthig Buch Verlag, Heidelberg 1992; b) A. Beil, D. Luckhaus, R. Marquardt, M. Quack, Faraday Dis. 1994, 99, 49; c) H. Grosenick, V. Schurig, J. Costante, A. André, Tetrahedron: Asym. 1995, 6, 87; d) Z. Jiang, J. Crassous, V. Schurig, Chirality 2005, 17, 488.

[16] M. Mazenauer, S. Manov, V. M. Galati, P. Kappeler, J. Stohner, $R S C$ Adv 2017, 7, 55434.

[17] a) L. P. H. Schmidt, T. Jahnke, A. Czasch, M. Schöffler, H. Schmidt-Böcking, R. Dörner Phys. Rev. Lett. 2012, 108, 73202; b) S. Zeller, M. Kunitski, J. Voigtsberger, A. Kalinin, A. Schottelius, C. Schober, M. Waitz, H. Sann, A. Hartung, T. Bauer, M. Pitzer, F. Trinter, C. Goihl, C. Janke, M. Richter, G. Kastirke, M. Weller, A. Czasch, M. Kitzler, M. Braune, R.E. Grisenti, W. Schöllkopf, L. P. H. Schmidt, M. S. Schöffler, J. B. Williams, T. Jahnke, R. Dörner, Proc. Nat. Acad. Sci. 2016, 113, 14651.

[18] a) M. Kunitski, S. Zeller, J. Voigtsberger, A. Kalinin, L. P. H. Schmidt, M. Schöffler, A Czasch, W. Schöllkopf, R. E. Grisenti, T. Jahnke, D. Blume, R. Dörner, Science 2015, 348,551 b) J. Voigtsberger, S. Zeller, J. Becht, N. Neumann, F. Sturm, H.-K. Kim, M. Waitz, F. Trinter, M. Kunitski, A. Kalinin, J. Wu, W. Schöllkopf, D. Bressanini, A. Czasch, J. B. Williams, K. Ullmann-Pfleger, L. P. H. Schmidt, M. S. Schöffler, R. E. Grisenti, T. Jahnke, R. Dörner, Nat. Commun. 2014, 5, 5765 .

[19] M. Pitzer, G. Kastirke, M. Kunitski, T. Jahnke, T. Bauer, C. Goihl, F. Trinter, C. Schober, K. Henrichs, J. Becht, S. Zeller, H. Gassert, M. Waitz, A. Kuhlins, A.S. Johnson, H. Sann, F. Sturm, F. Wiegandt, R. Wallauer, L. P. H. Schmidt, M. Mazenauer, B. Spenger, S. Marquardt, S. Marquardt, H. Schmidt-Böcking, J. Stohner, R. Dörner, M. S. Schöffler, R. Berger, ChemPhysChem 2016, 17, 2465.

[20] M. Pitzer, G. Kastirke, P. Burzynski, M. Weller, D. Metz, J. Neff, M. Waitz, F. Trinter, L. P. H. Schmidt, J. B. Williams, T. Jahnke, H. SchmidtBöcking, R. Berger, R. Dörner, M. Schöffler $J$. Phys. B: Opt. At. Mol. Phys. 2017, 49, 234001.

[21] K. Fehre, D. Trojanowskaja, J. Gatzke, M Kunitski, F. Trinter, S. Zeller, L. P. H. Schmidt, J. Stohner, R. Berger, A. Czasch, O. Jagutzki, T. Jahnke, R. Dörner, M. S. Schöffler, Rev. Sci. Instr. 2018, 89, 045112

[22] a) N. Böwering, T. Lischke, B. Schmidtke, N Müller, T. Khalil, U. Heinzmann, Phys. Rev Lett. 2001, 86, 1187; b) G. A. Garcia, L. Nahon, M. Lebech, J.-C. Houver, D. Dowek, I. Powis, J. Chem. Phys. 2003, 119, 8781; c) C. Lux, M. Wollenhaupt, T. Bolze, Q. Liang, J. Köhler, C. Sarpe, T. Baumert, Angew. Chem. Int. Ed. 2012, 51,5001

[23] M. Tia, M. Pitzer, G. Kastirke, J. Gatzke, H.-K. Kim, F. Trinter, J. Rist, A. Hartung, D. Trabert, J. Siebert, K. Henrichs, J. Becht, S. Zeller, H. Gassert, F. Wiegandt, R. Wallauer, A. Kuhlins, C. Schober, T. Bauer, N. Wechselberger, P. Burzynski, J. Neff, M. Weller, D. Metz, M. Kircher, M. Waitz, J. B. Williams, L. P. H. Schmidt, A. D. Müller, A. Knie, A. Hans, L. Ben Ltaief, A. Ehresmann, R. Berger, H. Fukuzawa, K. Ueda, H. Schmidt-Böcking, R. Dörner, J. Jahnke, P.V. Demekhin, M. Schöffler, J. Phys. Chem. Lett. 2017, 8, 2780.

[24] F. Trinter, L. P. H. Schmidt, T. Jahnke, M. S Schöffler, O. Jagutzki, A. Czasch, J. Lower, T. A. Isaev, R. Berger, A. L. Landers, T. Weber, R. Dörner, H. Schmidt-Böcking, Mol. Phys. 2012, $110,1863$.

[25] M. Pitzer, PhD Thesis, Goethe Universität Frankfurt, 2015. 